PSFC/JA-11-42

\title{
Surface thermocouples for measurement of pulsed heat flux in the divertor of the Alcator C-Mod tokamak
}

\author{
Brunner, D. and LaBombard, B.
}

February 2012

\section{Plasma Science and Fusion Center Massachusetts Institute of Technology Cambridge MA 02139 USA}

This work was supported by the U.S. Department of Energy, Grant No. DE-FC0299ER54512. Reproduction, translation, publication, use and disposal, in whole or in part, by or for the United States government is permitted. 


\title{
Surface thermocouples for measurement of pulsed heat flux in the divertor of the Alcator C-Mod tokamak
}

\author{
D. Brunner ${ }^{1}$ and B. LaBombard
}

MIT PSFC Cambridge, MA 02139 USA

A novel set of thermocouple sensors have been developed to measure heat fluxes arriving at divertor surfaces in the Alcator C-Mod tokamak, a magnetic confinement fusion experiment. These sensors operate in direct contact with the divertor plasma, which deposits heat fluxes in excess of $\sim 10 \mathrm{MW} / \mathrm{m}^{2}$ over a $\sim 1$ second pulse. Thermoelectric EMF is produced across a non-standard bimetallic junction: a $50 \mu \mathrm{m}$ thick $74 \%$ tungsten-26\% rhenium ribbon embedded in a $6.35 \mathrm{~mm}$ diameter molybdenum cylinder. The unique coaxial geometry of the sensor combined with its single-point electrical ground contact minimizes interference from the plasma/magnetic environment. Incident heat fluxes are inferred from surface temperature evolution via a 1-D thermal heat transport model. For an incident heat flux of $10 \mathrm{MW} / \mathrm{m}^{2}$, surface temperatures rise $\sim 1000{ }^{\circ} \mathrm{C} / \mathrm{s}$, corresponding to a heat flux flowing along the local magnetic field of $\sim 200 \mathrm{MW} / \mathrm{m}^{2}$. Separate calorimeter sensors are used to independently confirm the derived heat fluxes by comparing total energies deposited during a plasma pulse. Langmuir probes in close proximity to the surface thermocouples are used to test plasma-sheath heat transmission theory and to identify potential sources of discrepancies among physical models.

\section{Introduction}

The measurement and control of plasma-surface heat fluxes is a key issue for magnetic fusion devices. The performance of the core plasma is largely determined by the exposure of its first-wall components to energetic particles and radiation. Measurements of heat fluxes on the walls can, in turn, tell experimentalists about the rate of plasma transport that is occurring across magnetic field lines. In 2010, the U.S. Department of Energy established a Joint Research Target aimed a characterizing the 'heat flux footprints' in the divertor target regions of three U.S. tokamak experiments: NSTX, DIII-D and Alcator C-Mod. In preparation for this study, a new suite of thermal sensor diagnostics was developed and installed in Alcator C-Mod's outer divertor surfaces [1]. This paper discusses the design details and the performance characteristics of the surface thermocouple array that was installed in Alcator C-Mod-an array that proved to be a vital component of the overall diagnostic set.

Surface thermocouples (or 'self-renewing thermocouples' [2]) are different from standard thermocouples in that their thermojunction is formed in a very thin layer at the surface of the sensor. This is typically done by embedding two electrically insulated and dissimilar metallic wires into a carrier body and allowing them to come into electrical contact only at the very surface. This construction leads to a fast thermal time-response and is particularly useful if

\footnotetext{
${ }^{1}$ Author to whom correspondence should be addressed. Electronic mail: brunner@mit.edu
} 
one wants to characterize the surface temperature evolution of an actual component in service. In this case, one simply makes the carrier body out of the same material as the component under test. In addition, this construction also enables a simple computation of the incident heat flux to the component; the measured surface temperature evolution is applied as a boundary condition to a time-dependent thermal transport model of the component under test. In fusion plasmas, it is this latter quantity, the incident heat flux, which is most desired for assessing plasma thermal loads.

Originally developed to measure gun-bore surface temperatures [3], surface thermocouples have since been used for heat flux measurements in rocket nozzles [2,4], shock tunnels \& tubes [5,6], diesel engines [7], and reentrant vehicles [8]. A recent investigation of surface thermojunction formation and the subsequent time response is given in [9]. To our knowledge, Alcator C-Mod is the first and only use of surface thermocouples in a fusion reactor experiment. They were first tried in Alcator C-Mod the mid 1990's but were not used extensively due to poor signal quality $[10,11]$. We have since made substantial improvements to that original design, allowing them to be used for routine measurements in the C-Mod tokamak-a very harsh environment for such sensors.

\section{Design considerations for operation in a tokamak}

Surface thermocouples are typically made with two parallel ribbons to form the thermojunction. But this would necessitate bringing the signal out via twisted-pair leads, which is inferior to the use of a coaxial cable. In general, wire loops in low-voltage signal pathways must be minimized in a tokamak environment because, by Faraday's Law, time-varying magnetic fields induce voltage in the loops. Therefore, any transition from a two-ribbon sensor to a coaxial cable would problematic because of the small wire loop formed at the connection. Our surface thermocouples and cables all have a coaxial geometry to avoid this effect. The surface thermocouples are grounded at only one point to avoid ground loops.

There are strong currents (100's $\mathrm{kA} / \mathrm{m}^{2}$ parallel to the magnetic field) in the edge plasma of Alcator C-Mod that connect the inner and outer divertor [12]. The heat flux to the divertor surface is partially carried by these currents. Thus in order to insure that the surface thermocouple receives the same heat flux as the divertor surface, its body must be grounded to the divertor. Yet in the first version of the surface thermocouple sensor mount, it was found that the plasma-induced current caused a voltage on the same order of the thermal EMF. This is because the current flowed through the electrically resistive stainless steel holder to ground (see Figure 1). Propagation of this fictitious thermal EMF through the thermal analysis resulted in a large positive spike of heat flux at plasma initiation and a negative spike at plasma termination, making the measurements difficult to interpret. The present design electrically grounds the sensor through the molybdenum thermocouple body (via a 'copper ground collet' Figure 1 and Figure 2). Since the molybdenum body has a much smaller resistance than the stainless steel holder, the effect is essentially eliminated. With this proper ground placement, no negative heat fluxes are measured with the surface thermocouples (bottom panel of Figure 3). 
Since we are only interested in recording rapid changes in surface temperature from a plasma pulse, we do not need to select lead wires that match the Seebeck coefficients of the thermojunction metals. Thermal calibration of the entire circuit, with its various thermojunctions along the electrical pathway, would be impractical and is not needed. Instead, our scheme for measurements with surface thermocouples relies on all thermojunctions, except for that at the surface, to remain at constant temperature throughout the pulse. The most likely thermojunction to violate this assumption is the one between the surface thermocouple molybdenum body and its stainless steel holder. Using a semi-infinite body approximation [13] (the time for a temperature to change at a distance $x: t \approx x^{2} / 16 \alpha$, with $\alpha \approx 5.4$ $\times 10^{-5} \mathrm{~m}^{2} / \mathrm{s}$ for molybdenum and a surface thermocouple of length $x \approx 0.035 \mathrm{~m}$ ) the time scale for the back thermojunction to change by $1 \%$ is $\sim 1.4 \mathrm{~s}$, which is longer than the typical pulse flat-top of $1.0 \mathrm{~s}$. Thus the overall change in the sensor's EMF is due to that of the molybdenum/tungsten-rhenium thermojunction alone. It is only necessary to know the surface temperature before the plasma pulse in order to look-up the proper starting point on the voltage-to-temperature calibration curve.

\section{Surface thermocouple sensor design}

The surface thermocouples described in this report are part of a more extensive thermal diagnostics package (see Reference [1] for details) that is comprised of nine surface thermocouples, seven Langmuir probes, thirteen calorimeters, and ten tile thermocouples-all in view of an IR camera (Figure 4). The diagnostics are placed in a $12^{\circ}$ toroidal section of the lower, outer divertor, which is of greatest interest because it usually receives the largest heat flux. The magnetic field angle with respect to the divertor surface is typically less than $1^{\circ}$. The majority of plasma heat flux is carried along field lines, thus misalignments of tiles and divertor modules can cause magnetic shadowing of surfaces. To eliminate shadowing, the tiles and thermal sensors are ramped an additional $2^{\circ}$ into the magnetic field. This has the added benefit of increasing the heat flux signal. Placement of the diagnostics within the same $12^{\circ}$ toroidal sector removes uncertainties that arise in assuming toroidal symmetry.

The surface thermocouples are an adaptation of a standard product that is commercially available from NANMAC Corporation, the patented E12 series 'self-renewing thermocouple' [2]. However, unlike the two-ribbon design of the E12 product line, we requested that NANMAC build a set of sensors with only a single $74 \%$ tungsten- $26 \%$ rhenium ribbon ( $2 \mathrm{~mm}$ wide by $50 \mu \mathrm{m}$ thick) embedded into two molybdenum half-cylinders (Figure 2). This coaxial geometry is important because, as stated above, it minimizes EMF pickup from the tokamak's rapidly changing magnetic fields. Mica strips keep the ribbon electrically insulated from the molybdenum body. The assembly is held together by swaging it into a $6.35 \mathrm{~mm}$ outside diameter molybdenum cylinder.

Each surface thermocouple is bolted to the divertor structure through a custom stainless steel mount with a threaded, removable coaxial cable connector. The depth of the surface thermocouple body into the divertor is adjusted with a 
series of stainless steel shims until its face is just proud of the divertor tile surface. A diamond file is then used to file the surface thermocouple flush to the tile surface. This initiates the thermojunction between the tungstenrhenium and molybdenum by bending small slivers of molybdenum over the ribbon. We find that this thermojunction is reliably maintained, regardless of the plasma exposure conditions.

The surface thermocouple signal is carried on a standard $50 \Omega$ silicon-dioxide insulated coaxial cable through a ceramic-insulated coaxial vacuum feedthrough (Figure 1). The voltage signal, which is on the order of a few $\mathrm{mV}$, is processed by custom-built circuit boards. First it is differentially filtered with a simple R-C filter, $\tau_{\mathrm{RC}}=3.0 \mathrm{~ms}$, to attenuate high frequency noise. A high common-mode rejection differential amplifier (INA129) is then used to amplify the thermoelectric EMF by a factor of 248. Finally, the signal passes through a unity-gain amplifier (LF411A) to provide additional filtering $\left(\tau_{\mathrm{RC}}=3.0 \mathrm{~ms}\right)$ and to send the signal to a compact PCI data acquisition system. We employ a D-tAcq ACQ196 digitizer [14] operating at $400 \mathrm{kHz}$ sampling rates. Since there remains noise of order $\pm 50{ }^{\circ} \mathrm{C}$ (top panel of Figure 3) the temperature is digitally filtered with a time constant of $0.8 \mathrm{~ms}$ before input to thermal analysis.

NANMAC supplied a custom voltage-to-temperature calibration curve $\left(\sim 16 \mu \mathrm{V} /{ }^{\circ} \mathrm{C}\right)$ for the non-standard molybdenum/tungsten-rhenium thermojunction, which is valid over the range of 30 to $1600{ }^{\circ} \mathrm{C}$ and linearly extrapolated to higher temperatures. Before each plasma pulse the surface thermocouple voltage is calibrated using type- $\mathrm{K}$ thermocouples that are the part of the overall divertor diagnostic package. In particular, we use the thermocouple embedded in the divertor tile that corresponds to the surface thermocouple. These ice-point compensated junctions measure the absolute temperature of each tile to within an accuracy of $2{ }^{\circ} \mathrm{C}$. Since Alcator CMod operates with a $\sim 20$ minute time delay between plasma pulses, there is more than sufficient time for the surface thermocouples, their surrounding divertor tiles, and embedded type-K thermocouples to achieve thermal equilibrium by the start of the following plasma pulse.

During a typical plasma pulse, the 'footprint' of the plasma-surface heat flux is interrogated in detail by slowly sweeping it (via programmed changes in the plasma's magnetic equilibrium) over the surface thermocouple sensor array. This produces time traces of surface temperature, plasma flux surface position, and magnetic field angle for a given sensor, such as those shown in the top three panels of Figure 3. Heat flux is calculated using QFLUX_1D, a one-dimensional, finite element thermal analysis code with temperature dependent thermal properties that allows for different materials, cross sectional areas, and lengths for each volume element. The surface temperature time history is imposed as the front-surface boundary condition. Energy loss due to surface thermal radiation is neglected because it is much less than the incident plasma heat flux $\left(q_{\text {radiation }} \sim 10^{4} \mathrm{~W} / \mathrm{m}^{2}\right.$ at $\sim 1000{ }^{\circ} \mathrm{C}$ compared to $q_{\text {plasma }} \sim 10^{7}$ $\mathrm{W} / \mathrm{m}^{2}$ ). A zero heat flux boundary condition is applied to the back surface since the thermocouple body is essentially semi-infinite on the time scale of the pulse. The time evolution of the magnetic field in the tokamak, and in particular at the sensor locations, is derived from the EFIT plasma equilibrium code [15]. This output allows the equivalent heat flux flowing along magnetic field lines to be computed (bottom panel in Figure 7) along with the 
mapping of that field line into the scrape-off layer, as it passes the outer equatorial mid-plane ( $\rho$-coordinate in third panel of Figure 7 with the location of the last-closed flux surface corresponding to $\rho=0$ ).

\section{Performance}

The three largest sources for error in the surface thermocouple electrical signal are: 1) voltage pick-up from large, time-varying magnetic fields, 2) temperature changes at other thermojunctions in the circuit during the plasma pulse, and 3) voltages generated by plasma-induced currents flowing to ground through resistive sensor leads. These effects have been largely minimized by the sensor's design, as can be assessed by studying the behavior of the sensor signals. However, we have found that the surface thermocouple does have some important operational limitations that must be observed.

Observations that the surface thermocouple is operating properly may be made through comparison of measurements with and without plasma contact (Figure 5). Unless the surface thermocouple is directly exposed to plasma contact, the sensor voltage remains relatively constant in time over the period when thermal analysis is performed. This demonstrates that the changing magnetic fields associated with routine tokamak operation do not affect the surface thermocouple and that only the surface thermojunction is changing temperature. The lack of a temperature change at the thermojunction between the surface thermocouple and its stainless steel mount is also confirmed by the QFLUX_1D thermal simulations that use the surface temperature to calculate heat flux.

We have found that voltage pick-up during Ion-Cyclotron Range of Frequencies (ICRF) heating in Alcator C-Mod [16] precludes the use of surface thermocouples. Small levels of voltage pick-up are seen when ICRF antennas are turned to $80 \mathrm{MHz}$. The pick-up voltage increase when the antennas are turned to $50 \mathrm{MHz}$. Fortunately, there is no pick-up seen during operation of lower hybrid heating or lower-hybrid current drive [16] (both at $4.6 \mathrm{GHz}$ ), allowing surface thermocouple analysis to be performed in these discharges. Measurements of surface heat flux during a plasma disruption, i.e. a rapid loss of plasma confinement [17], would be of great interest. In these events, the plasma stored energy $(\sim 100 \mathrm{~kJ})$ is transferred from the plasma to the wall in $\sim 1 \mathrm{~ms}$. Unfortunately we have found that the voltage pick-up during a disruption typically overwhelms the thermal signal.

\section{Comparison with other heat flux diagnostics}

Utilizing the extensive array of sensors installed on C-Mod's outer divertor (Figure 4), it is possible to perform a number of comparisons among the surface heat flux diagnostics. One important crosscheck is with the calorimeter sensors. These are thermally isolated molybdenum slugs with ice-point compensated, type- $\mathrm{K}$ thermocouples embedded in them at a location that is located approximately $5 \mathrm{~mm}$ from the calorimeter surface [18]. Because the thermojunction is recessed below the surface, the calorimeter signals have a time response that is nearly identical to that of the embedded tile thermocouples (top panel of Figure 3). This signal cannot be used to infer surface heat 
fluxes directly. Yet, because the sensors are thermally isolated, their overall change in temperature is a reliable measure of the time-integrated surface heat flux density. This quantity is computed for each calorimeter, accounting for its mass and exposed surface area. For comparison, a corresponding time-integration of the surface heat flux signal derived from the surface thermocouple data is performed. It is found that the surface thermocouple-derived and calorimeter-derived energy flux densities agree within 15\% (Figure 6).

A direct comparison of heat flux time histories derived from the surface thermocouple data can be made from Langmuir probes that are located in close proximity (Figure 4). The theory for heat flux through a plasma-wall sheath is well developed [19]:

$$
q=\gamma k_{B} T_{e} J_{\text {sat }} .
$$

With the electron temperature $\left(T_{e}\right)$ and particle flux density $\left(J_{\text {sat }}\right)$ measured by a Langmuir probe swept through ionsaturation; $k_{B}$ is Boltzmann's constant. The sheath heat flux transmission coefficient $(\gamma)$ is given by

$$
\gamma=2.5 \frac{T_{i}}{Z T_{e}}+\frac{2}{1-\delta_{e}}\left(1-\frac{J_{g n d}}{J_{s a t}}\right)+\ln \left[\frac{1-\delta_{e}}{\sqrt{2 \pi \frac{m_{e}}{m_{i}}\left(Z+\frac{T_{i}}{T_{e}}\right)}\left(1-\frac{J_{g n d}}{J_{s a t}}\right)}\right]
$$

Where $m_{i}$ and $m_{e}$ are the ion and electron masses, respectively; $Z$ is the ion charge. This expression accounts for the non-zero plasma-driven current density $\left(J_{\text {gnd }}\right)$ that flows to the divertor, i.e. the ground current. Positive current corresponds to net ion collection by the divertor. The ground current is measured as the current density to a Langmuir probe when it is held at zero bias with respect to the divertor. Both the ion temperature $\left(T_{i}\right)$ and the secondary electron emission coefficients $\left(\delta_{e}\right)$ are not measured. Here, the ion temperature is taken to be equal to the electron temperature, although it can be a factor of $\sim 2$ greater in the edge of tokamaks [20]. Secondary electron emission is assumed to be zero. The small grazing angle of the magnetic field line with respect to the surface $\left(\sim 2^{\circ}\right)$ makes prompt recapture likely [19]. For a deuterium plasma these assumptions $\left(T_{i}=T_{e}\right.$ and $\left.\delta_{e}=0\right)$ along with a floating surface $\left(J_{\text {gnd }}=0\right)$ result in the heat transfer coefficient that is customarily assumed, $\gamma \approx 7.5$.

It should be pointed out that there has been difficulty successfully matching sheath heat flux measurements with theory in tokamaks (see Reference [21] and those within). Previous measurements at C-Mod, comparing data from an IR camera with Langmuir probes, also did not follow theory [22]. But, this investigation compared data from two different locations separated toroidally by $90^{\circ}$; shadowing of the Langmuir probes from divertor cassette misalignments may have played a role. However, the new experimental arrangement shown in 
Figure 4 avoids this complication. Comparison of adjacent surface thermocouples and Langmuir probes in the low density sheath-limited regime yields excellent agreement with sheath theory (Figure 7). These results show that a floating surface assumption $\left(J_{\text {gnd }}=0\right)$ is not appropriate for a grounded divertor. The ground current increases the heat flux by nearly $50 \%$, almost consistently through the whole SOL.

Yet we find that this agreement does not hold in all edge plasma regimes. As the density, and thus collisionality, is increased, the experimentally measured heat flux transmission coefficient decreases. Just before the onset of divertor detachment (i.e., a loss of plasma pressure along magnetic field lines) the experimentally measured heat transfer coefficient decreases below the minimum theoretically allowed value of $\gamma \approx 5$ (when $T_{i} \ll T_{e}$ ). Coincident with this behavior is the detection of excess plasma pressure $(\sim 2 \times)$ on divertor Langmuir probes relative to those upstream-a situation seen before on C-Mod called the 'death-ray' [12]. At that time the 'death-ray' was discovered C-Mod lacked thermal measurements in the divertor; it was speculated that the divertor over-pressure was caused by ionization of neutrals flowing with large toroidal velocities (since ion-neutral momentum coupling is very strong in this regime), depositing their momentum on magnetic flux surfaces that, for some unknown reason, had slightly elevated temperatures $\left(T_{e}>5 \mathrm{eV}\right.$, ionization dominant) relative to the rest of the divertor profile $\left(T_{e}<5 \mathrm{eV}\right.$, chargeexchange dominant). Moreover, the phenomenon was assumed to occur in an axis-symmetric band, affecting the whole divertor. But with the new surface thermocouple sensors installed on C-Mod, we now see that the 'death-ray' is not an axis-symmetric effect. Instead, it is likely restricted to just the magnetic flux tube intercepted by the

Langmuir probe. Apparently, the Langmuir probe's negative bias modifies the plasma in the flux tube. In this situation the parallel electron heat loss may be blocked by the negative bias, causing the local flux tube to be elevated above the nominal $T_{e} \approx 5 \mathrm{eV}$ threshold for strong ionization [23]. By this effect, neutral momentum transfer may still cause the local over-pressure, but only on the flux tube intercepted by the biased Langmuir probe. Prompted by these measurements, this phenomenon is now being studied in detail with the help of the UEDGE plasma transport simulation code [24]. In any case, the new surface thermocouple sensors have proven their worth in identifying regimes where divertor Langmuir probes accurately measure local plasma parameters in C-Mod. This has allowed for a proper comparison of divertor and upstream plasma measurements, leading to the important result that the divertor heat flux footprint is largely set by the upstream plasma pressure profile [1].

\section{Summary}

We have developed surface thermocouple sensors for use in a tokamak plasma environment and investigated their performance in the Alcator C-Mod experimental device. These surface thermocouples have been successfully used to infer the heat flux densities arriving on the outer divertor target. It has been demonstrated that the sensor's voltage response during a plasma pulse accurately reports the change in divertor surface temperature. Energy fluxes computed from the surface thermocouples are independently confirmed with calorimeters. Comparisons with Langmuir probe derived heat fluxes yield good agreement in low density regimes, lending confidence to the sensor's measurements and plasma-sheath theory. The surface thermocouples have also identified regimes where further 
analysis is needed for proper interpretation of the Langmuir probe data. In light of these favorable results, surface thermocouple sensors will continue to be employed in C-Mod as an important part of the divertor diagnostics set.

\section{Acknowledgments}

We would like to thank the entire Alcator C-Mod team, especially Ron Rosati for his craftsmanship in preparing the thermocouples and cables. Special thanks are extended to Tom Blanchard and the team at NANMAC Corporation for supplying the custom-designed 'self-renewing thermocouple' sensors. D.B. would like to thank Adam McLean for useful discussions. Work supported by US DoE Coop. Agreement DE-FC02-99ER54512.

\section{References}

[1] B. LaBombard, J. L. Terry, J. W. Hughes, D. Brunner, J. Payne, M. L. Reinke, I. Cziegler, R. Granetz, M. Greenwald, I. H. Hutchinson, J. Irby, Y. Lin, B. Lipschultz, Y. Ma, E. S. Marmar, W. L. Rowan, N. Tsujii, G. Wallace, D. G. Whyte, S. Wolfe, S. Wukitch, G. Wurden, and the Alcator C-Mod Team, Physics of Plasmas 18, (2011).

[2] J. Nanigian and D. Nanigian, Proceedings of SPIE 6222, p. 622203, (2006).

[3] D.A. Benderskey, Mechanical Engineering 75, 117, (1953).

[4] C.E. Moeller, M. Noland, and B.L. Rhodes, NASA Contributions to Development of Special Purpose Thermocouples, A Survey, NASA SP-5050, pp. 37-44, (1968).

[5] V. Menezes and S. Bhat, Review of Scientific Instruments 81, 104905 (2010).

[6] H. Mohammed, H. Salleh, and M. Yusoff, International Communications in Heat and Mass Transfer 35, 853, (2008).

[7] A. Alkidas and R. Cole, Journal of Heat Transfer 107, 439, (1985).

[8] C. Kidd, Measurement Technology for Aerospace Applications in High-Temperature Environments 1, pp. 3150, (1992).

[9] D.R. Buttsworth, Experimental Thermal and Fluid Science 25, 409, (2001).

[10] S. Gangadhara, B. LaBombard, B. Lipschultz, and N. Pierce, in Bulletin of the American Physical Society, 41, 1550, (1996).

[11] N. Basse, A. Domingue, E.M. Edlund, C.L. Fiore, R.S. Grantez, A.E. Hubbard, J.W. Hughes, I.H. Hutchinson, J.H. Irby, B. LaBombard, L. Lin, Y. Lin, B. Lipschultz, J.E. Liptac, E.S. Marmar, D.A. 
Mossessian, R.R. Parker, M. Porkolab, J.E. Rice, J A. Snipes, V. Tang, J. L. Terry, S. J. Wolfe, and K. Zhurovich, Fusion Science and Technology 51, 476, (2007).

[12] B. LaBombard, J. Goetz, I. H. Hutchinson, D. Jablonski, J. Kesner, C. Kurz, B. Lipschultz, G. McCracken, A. Niemczewski, J. L. Terry, A. Allen, R. Boivin, F. Bombarda, P. Bonoli, C. Christensen, C. Fiore, D. Garnier, S. Golovato, R. Granetz, M. Greenwald, S. Horne, A. Hubbard, J. Irby, D. Lo, D. Lumma, E. Marmar, M. May, A. Mazerenko, R. Nachtrieb, H. Ohkawa, P. O'Shea, M. Porkolab, J. Reardon, J. Rice, J. Rost, J. Schachter, J. Snipes, J. Sorci, P. Stek, Y. Takase, Y. Wang, R. Watterson, J. Weaver, B. Welch, and S. Wolfe, Journal of Nuclear Materials 241, 149, (1997).

[13] A. F. Mills, Heat Transfer. (Homewood, IL: Irwin Publishing, 1992).

[14] D-tAcq Solutions Ltd., East Kilbride, Scotland, UK, http://www.d-tacq.com/.

[15] L.L. Lao, H. St. John, R.D. Stambaugh, A.G. Kellman, and W. Pfeiffer, Nuclear Fusion 25, 1611, (1985).

[16] P.T. Bonoli, R. Parker, S.J. Wukitch, Y. Lin, M. Porkolab, J.C. Wright, E. Edlund, T. Graves, L. Lin, J. Liptac, A. Parisot, A.E. Schmitt, V. Tang, W. Beck, R. Childs, M. Grimes, D. Gwinn, D. Johnson, J. Irby, A. Kanojia, P. Koert, S. Marazita, E. Marmar, D. Terry, R. Vieira, G. Wallace, J. Zaks, S. Barnabei, C. Brunkhorse, R. Ellis, E. Fredd, N. Greenough, J. Hosea, C.C. Kung, G.D. Loesser, J. Rushinski, G. Schilling, C.K. Phillips, J.R. Wilson, R.W. Harvey, C. Fiore, R. Granetz, M. Greenwald, A.E. Hubbard, I.H. Hutchinson, B. LaBombard, B. Lipschultz, J. Rice, J.A. Snipes, J. Terry, S.M. Wolfe, and the Alcator C-Mod Team, Fusion Science and Technology 51, 3, pp. 401-436, (2007).

[17] M. Sugihara, M. Shimada, H. Fujieda, Yu. Gribov, K Ioki, Y. Kawano, R. Khayrutdinov, V. Lukash, and J. Ohmori, Nuclear Fusion 47, 337-352, (2007).

[18] J. Payne, D. Brunner, B. LaBombard, and J. Terry, "Divertor calorimetery in Alcator C-Mod," Review of Scientific Instruments, to be submitted.

[19] P. C. Stangeby, The plasma boundary of magnetic fusion devices. (New York, NY: Taylor and Francis Group, 2000).

[20] M. Kocan, J. Gunn, J. Pascal, and E. Gauthier, Contributions to Plasma Physics 50, 836, (2010).

[21] J. Marki, R. Pitts, T. Eich, A. Herrmann, J. Horacek, F. Sanchez, and and G. Veres, Journal of Nuclear Materials 363, 382, (2007).

[22] D. Brunner, B. LaBombard, J. Payne, and J.L. Terry, Journal of Nuclear Materials 415, (2011).

[23] D. Brunner, B. LaBombard, J.L. Terry, and and M. Reinke, in Bulletin of the American Physical Society, 55, (2010).

[24] M. Umansky, D. Brunner, B. LaBombard, and T. Rognlien, "Modeling of local edge plasma perturbations 
induced by a biased," in Proceedings of PET, (2011), submitted. 


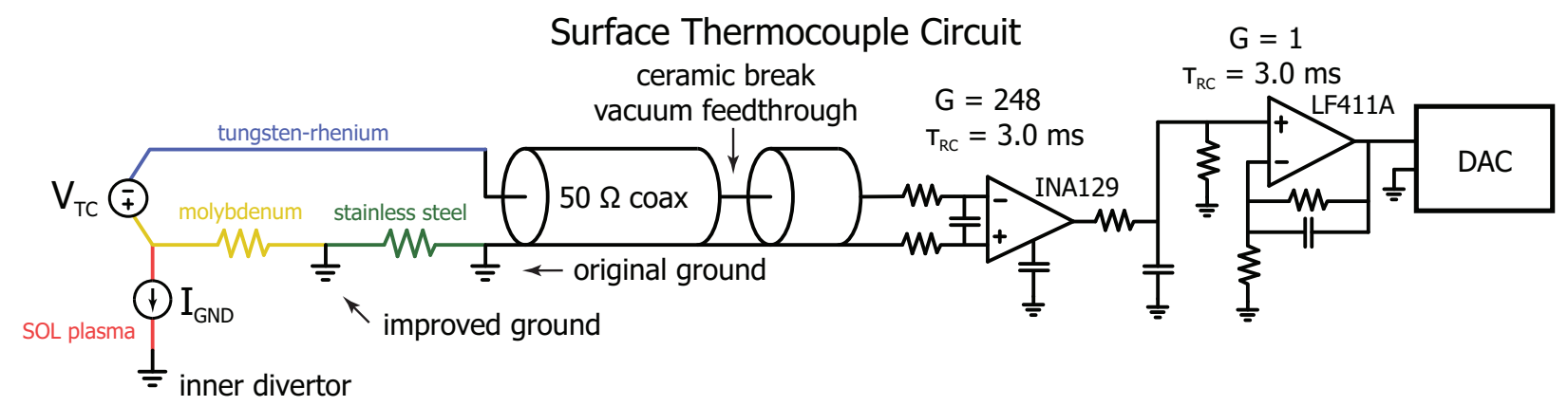

Figure 1. (color online) The thermal EMF $\left(\mathrm{V}_{\mathrm{TC}}\right)$ is generated at the molybdenum/tungsten-rhenium thermojunction.

No other thermojunctions change temperature through the pulse. The voltage is transmitted via a $50 \Omega$ coax to a

high common-mode rejection instrumentation amplifier with a gain of 248. The differential input RC filter has a 3.0 $\mathrm{ms}$ time constant. The signal is put through a unity gain circuit with further filtering ( $3.0 \mathrm{~ms}$ time constant) and then digitized on a D-TacQ DT196 unit at $400 \mathrm{kHz}$. Both the original and improved grounding points are shown (ground taken to be the vacuum vessel potential). With the old grounding method, plasma-induced currents would flow though the stainless steel thermocouple holder, contributing a voltage that was of the same order and sign of $\mathrm{V}_{\mathrm{TC}}$, leading to erroneous temperature measurements. In the present design the current path through the stainless steel is eliminated and the molybdenum body is shorted directly to ground. The voltage generated by the plasma-induced current flowing through the molybdenum is much less than $\mathrm{V}_{\mathrm{TC}}$. 


\section{Surface Thermocouple Assembly}

1. tungsten-rhenium ribbon

2. mica isolators

3. molybdenum half-cylinders

4. molybdenum full-cylinder

5. molybdenum tile

6. stainless steel divertor plate

7. copper ground collet

8. collet pusher

9. mica ground isolator
10. stack of shim-stock spacers

11. thermocouple holder

12. set screws

13. ceramic isolating spacers

14. center conductor pin

15. holder screw

16. Belleville washer

17. ceramic insulators
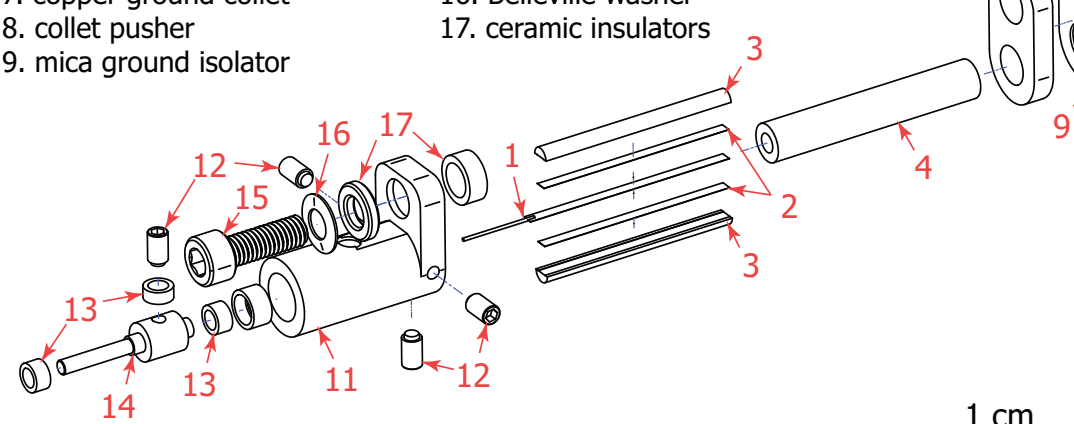

9

$1 \mathrm{~cm}$

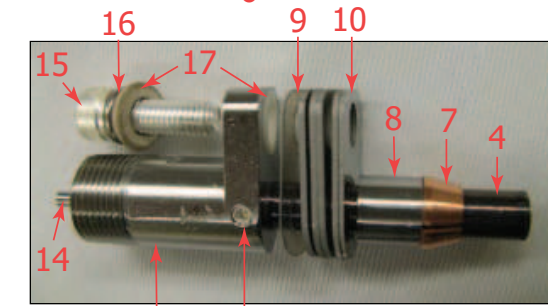

$11 \quad 12$

Figure 2. (color online) The $1 \mathrm{~cm}$ scale bar is for both the line drawing and inset photograph. The $74 \%$ tungsten$26 \%$ rhenium ribbon (1) is electrically isolated with mica sheets (2) from the molybdenum probe body ( $3 \& 4)$. This in turn is swaged into a 6.35 diameter molybdenum tube; forming a customized version of a 'self-renewing thermocouple' supplied by NANMAC Corp. Filing the surface of the sensor initiates the thermojunction. The copper collet (7) grounds the molybdenum senor body to the molybdenum tile with low electrical resistance. Insulators $(9 \&$ 13) isolate the stainless steel probe body from ground. The threaded holder (11) allows for a removable coaxial connection. 


\section{Temperature and Heat Flux from}

Strike Point Sweep

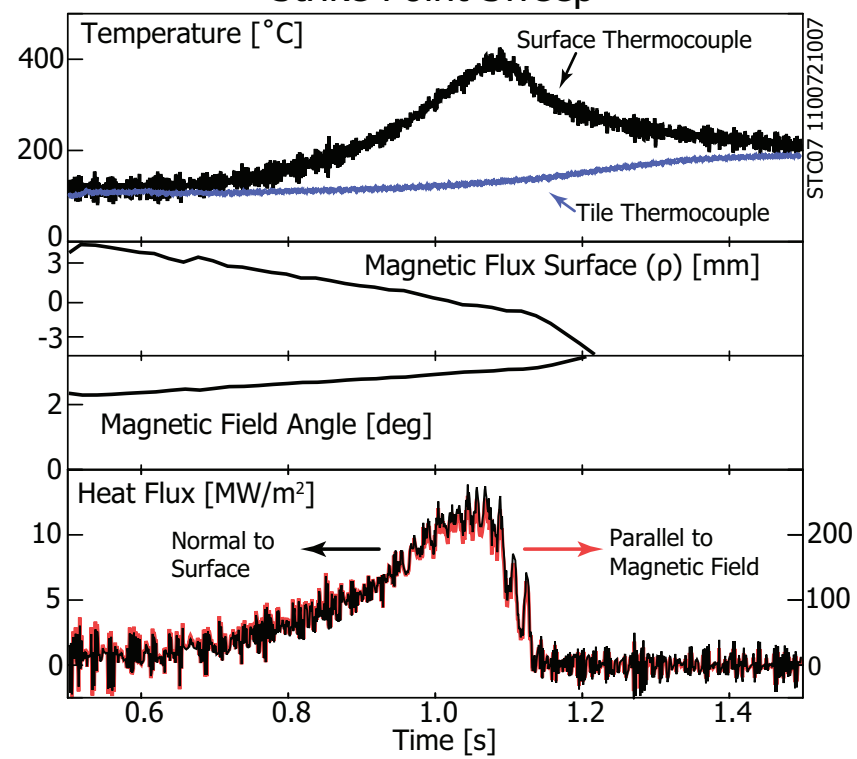

Figure 3. (color online) A cross-calibration procedure is performed on each discharge, which forces the surface thermocouple temperature to agree with the tile thermocouple temperature before the plasma pulse. The time evolution of both signals is shown in the top panel. Using output from the EFIT plasma equilibrium reconstruction code, the magnetic flux surface label $(\rho)$ and magnetic field line inclination angle on each surface thermocouple is recorded (second and third panel). The coordinate $\rho$ is the distance of the magnetic flux surface beyond the lastclosed flux surface as it passes the outer equatorial mid-plane of the plasma. Local heat flux density normal to the surface thermocouple surface is computed from a 1-D heat transport model of the surface thermocouple cylinder with temperature-dependent thermal parameters. This quantity and its equivalent heat flux density flowing along magnetic field lines are shown in the bottom panel. 


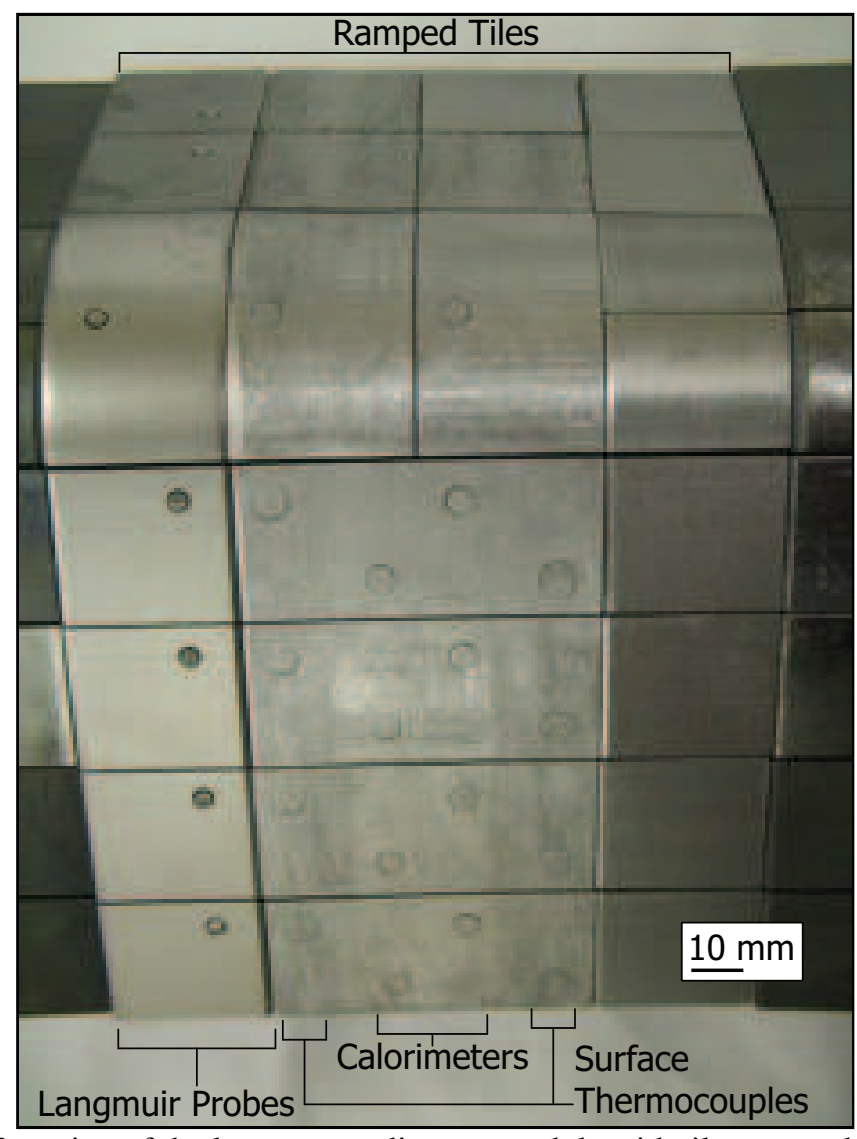

Figure 4. (color online) $12^{\circ}$ section of the lower outer divertor module with tiles ramped $2^{\circ}$ and sensors. The magnetic field is nearly tangential to the four lowest rows $\left(\sim 1^{\circ}\right.$ for the normal tiles, $\sim 2^{\circ}$ for the ramped). The Langmuir probes are the circles in the left-most column of tiles. The surface thermocouples are the left and rightmost circles in the center column of tiles. The calorimeters are the two columns of circles running in between the surface thermocouples. 


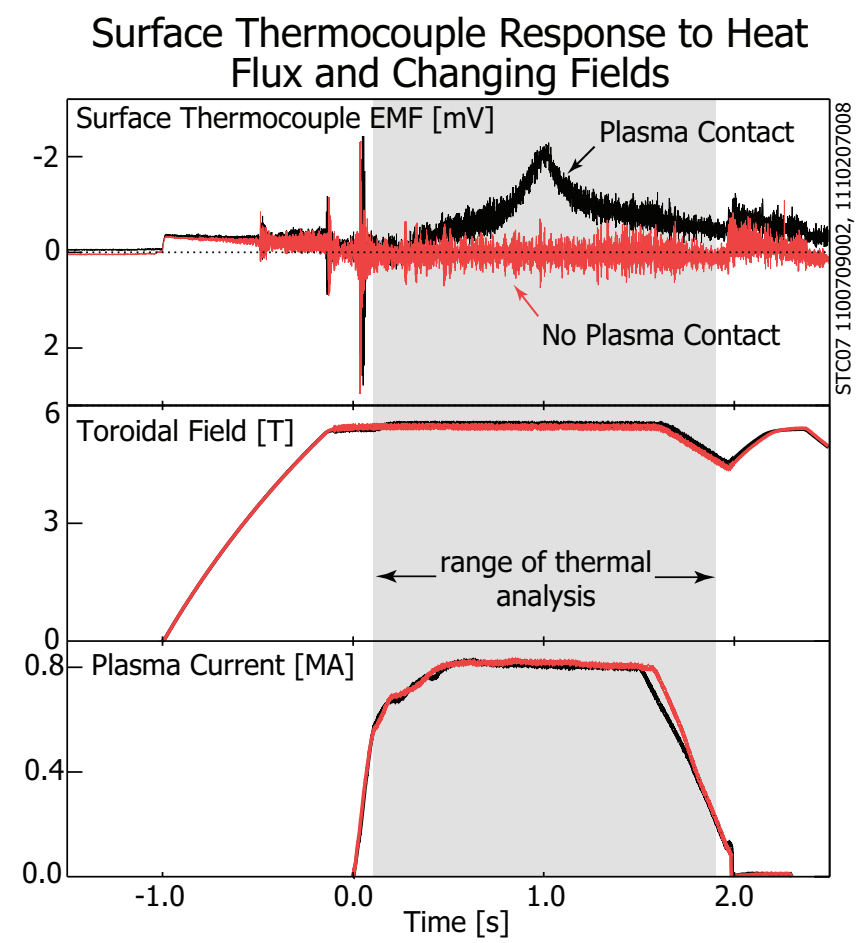

Figure 5. (color online) Comparison of raw surface thermocouple voltages for a pulse with plasma contacting the surface thermocouple sensor and one without (top panel). Note that using the tungsten-rhenium as the center conductor results in a decrease in voltage representing an increase in temperature. Vertical axis is inverted for clarity. Both pulses have nearly identical toroidal field (middle panel) and plasma current (bottom panel). There is a small sensitivity to the rate of toroidal field ramp-up and ramp-down, but neither affects the thermal analysis. The heat flux calculation starts after the toroidal field ramp-up induced voltage returns to its starting value and ends before the ramp-down. The sensor does not pick up any voltage contribution from plasma current ramps, as seen by the lack of a response during the case with no plasma contact. This lack of voltage change also indicates that only the surface thermojunction, and none of the others in the circuit, changes its temperature over a plasma pulse. From this comparison we are confident that the voltage response of the sensor is entirely due to changes in its surface temperature. 


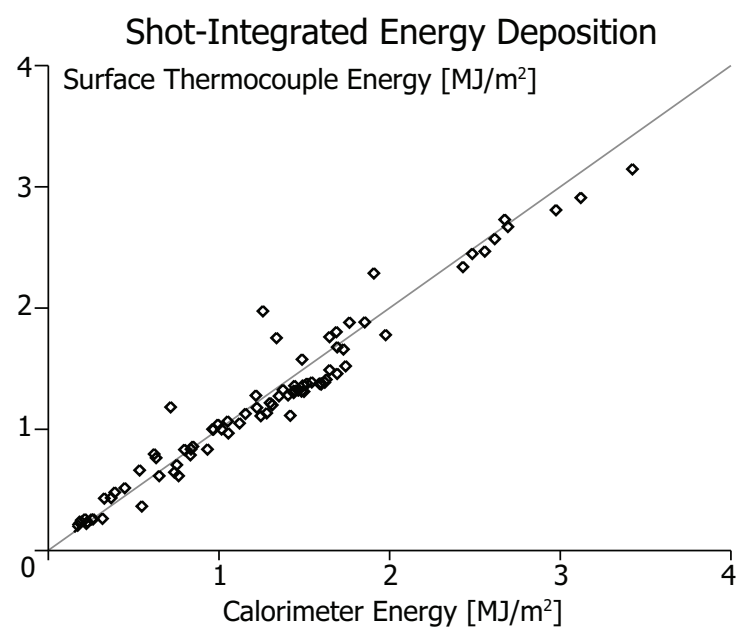

Figure 6. Comparison of the energy deposited on a surface thermocouple and neighboring calorimeter for all of the L-Mode pulses taken for the 2010 Joint Research Target [1]. Calorimeter energy flux is calculated using the temperature difference of a thermally isolated molybdenum slug with an embedded thermocouple. Surface thermocouple energy flux is found by integrating the surface heat flux, which was calculated from the surface temperature evolution. Values typically agree to better than $15 \%$. 


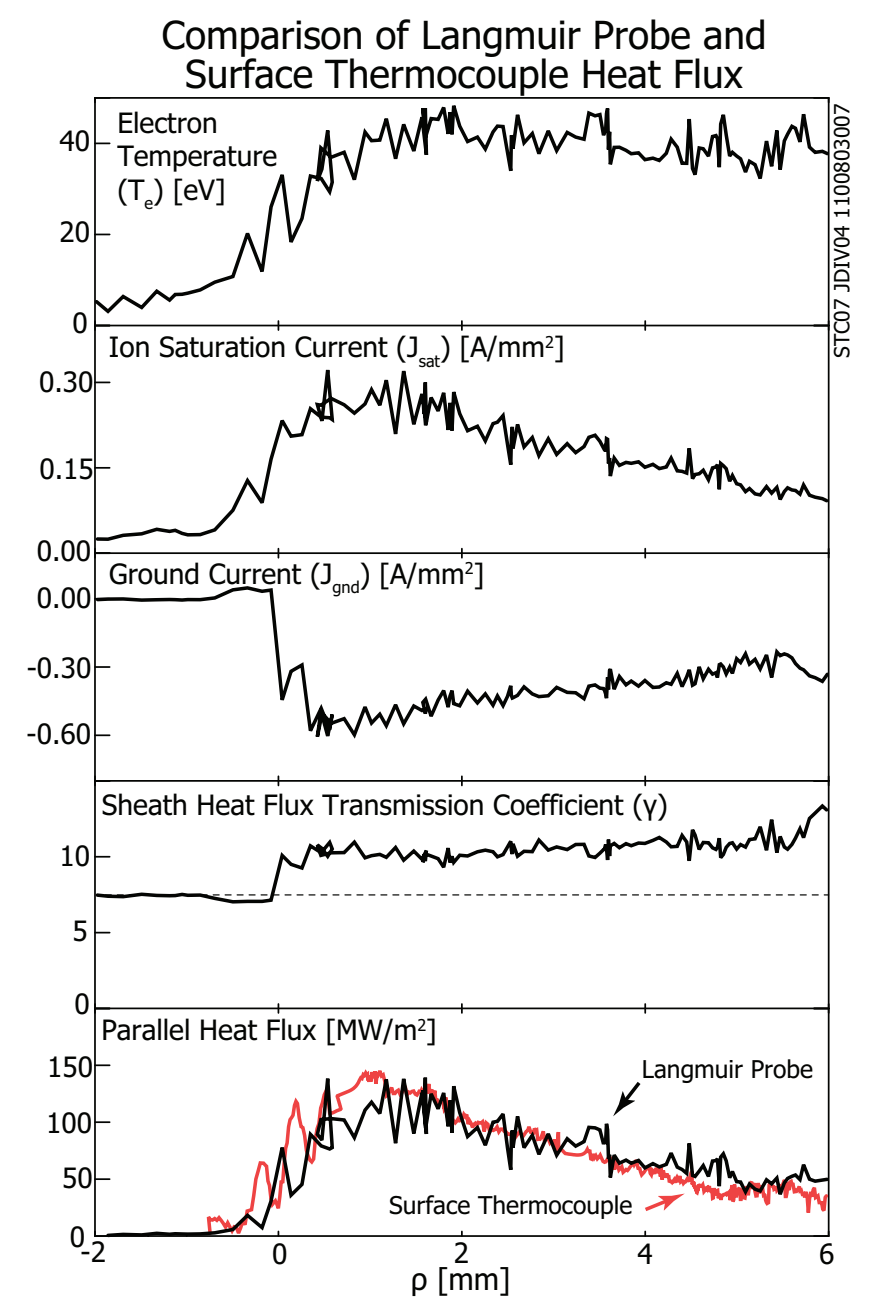

Figure 7. (color online) Plasma parameters and derived parallel heat flux density profiles obtained from sweeping the 'heat flux footprint' over a surface thermocouple and Langmuir probe in close proximity. Horizontal axis is $\rho$, the magnetic flux surface label, mapped to the outer midplane. The plasma-induced ground current $\left(J_{\text {gnd }}\right)$ at the outer divertor increases the sheath heat flux coefficient to $\sim 11,50 \%$ above the nominal floating value of $\sim 7.5$ (dashed line). Using this calculated value of the sheath heat flux transmission coefficient, the heat flux profiles from the Langmuir probe and surface thermocouple are in good agreement across the entire footprint. 